\title{
Towards TEM Quantification of Quantum Dot Dispersions in Cell Growth Media and In Vitro Cellular Uptake
}

\author{
N.S. Hondow, ${ }^{*}$ A. Brown, ${ }^{*}$ R. Brydson, ${ }^{*}$ P. Wang, ${ }^{* *}$ M.D. Holton, ${ }^{* * *}$ P. Rees, ${ }^{* * *}$ and H.D. \\ Summers*** \\ * Institute for Materials Research, University of Leeds, Leeds, LS2 9JT, UK \\ ** Institute of Molecular and Cellular Biology, University of Leeds, Leeds, LS2 9JT, UK \\ *** Centre for Nanohealth, College of Engineering, Swansea University, Swansea, SA2 8PP, UK
}

Engineered nanoparticles are finding applications in fields as varied as healthcare and energy generation. Nanotoxicology and nanomedicine are both concerned with the delivery of nano-objects to human cells, whether this be unintentional exposure by way of, for example, occupation or cosmetics (nanotoxicology) or by design, such as drug-delivery vehicles or magnetic resonance imaging contrast agents (nanomedicine). There are a rapidly growing number of in vitro studies involving the dosing of cell lines with nanoparticles, with a range of conclusions regarding toxicity outcomes, yet the key factors affecting nanoparticle uptake are not firmly established, e.g. [1,2].

Nanoparticles that are used in vitro are often coated with polymers or proteins to facilitate entry into cells and one property that can affect uptake is the aggregation or dispersion state. This is often well characterised in simple solutions by light scattering techniques yet when delivered to cell lines nanoparticles are dispersed in solutions of biological media and serum where, because of interaction with and scattering from the (protein) media, the particle dispersion is less well characterized [3].

This study has investigated the uptake by U2-OS human osteosarcoma cells of CdTe/ZnS core/shell quantum dots dispersed in biological media and serum. Quantitative cell (optical) imaging has been used to show that the nanoparticle uptake into cellular vesicles proceeds by a random process [4]. This was achieved by assuming groups of quantum dots within vesicles act as discrete and stable fluorescent sources. We have prepared standard thin sections of these cells for correlation with the optical results. TEM imaging can quantify the number of quantum dot loaded vesicles per cell section (Fig. 1a) and the number of dots per vesicle (Fig. 1b).

Of particular interest is correlation of the size of the quantum dot loaded vesicles with the size distribution of aggregates present in the cell growth media and serum. There is emerging evidence that uptake pathways are very dependent on the aggregate size of the nanoparticles [5]. As already discussed, assessing the nanoparticle dispersion in the biological media and serum is not trivial, dynamic light scattering and nanoparticle tracking analysis suggest aggregate sizes in the range of $\sim 8$ $\mathrm{nm}$ to more than $1000 \mathrm{~nm}$. TEM imaging has been used here to isolate the nanoparticle fraction of this distribution.

Conventional TEM, with samples prepared by simply dropcasting suspensions onto a thin carbon film, enables imaging of individual nanoparticles but, because of the drying process, is not representative of the particle aggregation in the dispersion. To overcome this problem we have prepared thin sections of nanoparticle solutions for TEM by plunge-freezing a blotted grid into liquid ethane [6]. The samples are then kept frozen in liquid nitrogen and examined by cryo-TEM. This standard biological sample preparation route allows the TEM imaging of aggregates of dots as they 
exist in solution (Fig. 2a). As the grid is warmed to room temperature we show that the nanoparticle dispersion remains stable (Fig. 2b). Devitrification causes little displacement of macromolecules [4], and consequently the nanoparticle distribution and hence aggregation state on the grid is unaltered by warming. This facilitates easy quantification of the nanoparticles dispersion.

\section{References}

[1] N. Singh et al., Biomaterials 30 (2009) 3891.

[2] H.F. Krug and P. Wick, Angew. Chem. Int. Ed. 50 (2011) 1260.

[3] S.H. Doak et al., Mutagenesis 24 (2009) 285.

[4] H.D. Summers et al., Nat. Nanotechnol. doi:10.1038/NNANO.201.277

[5] M. Motskin et al., Biomaterials 30 (2009) 3307.

[6] H.D. White et al., J. Struct. Biol. 121 (1998) 306.

[7] J. Dubochet in Cellular Electron Microscopy (Ed. J.R. McIntosh), Elsevier, London, 2007.

(a)

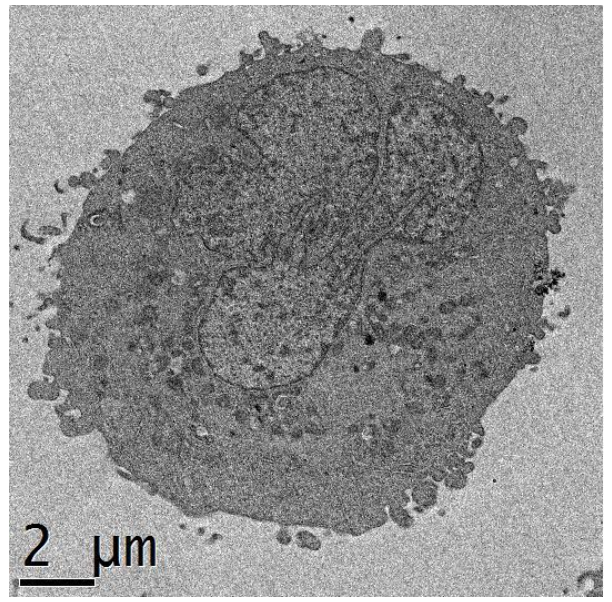

(b)

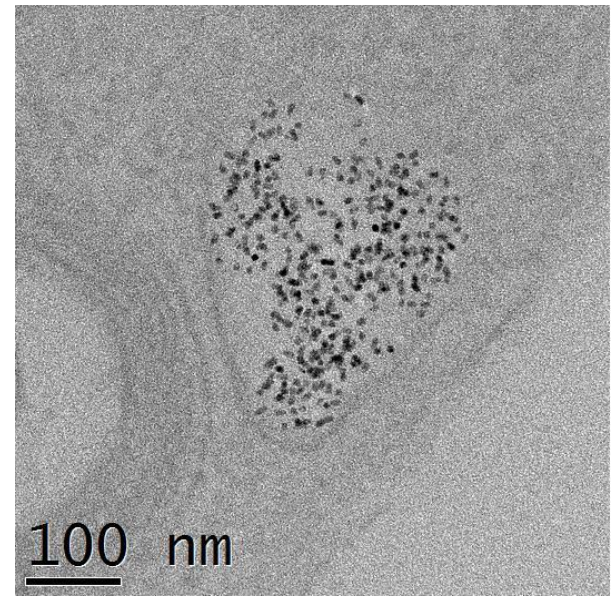

Fig.1. (a) Bright Field TEM image of a U2-OS cell with 17 vesicles containing quantum dots (from 2 to more than 1000 quantum dots). (b) Higher magnification image of one of the vesicles containing quantum dots.

(a)

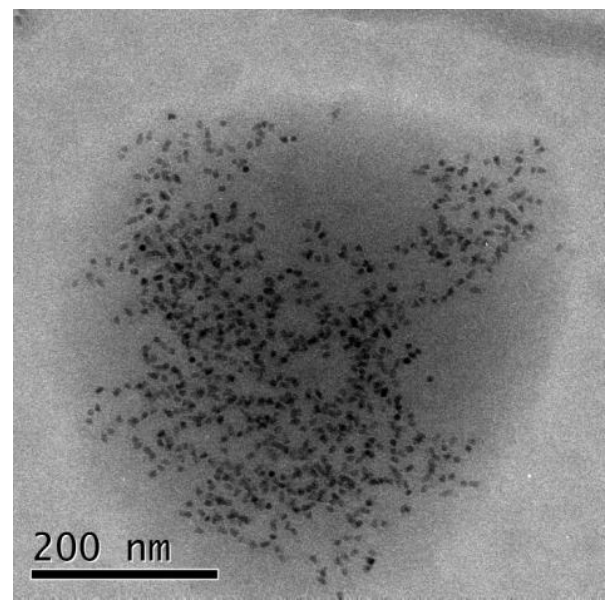

(b)

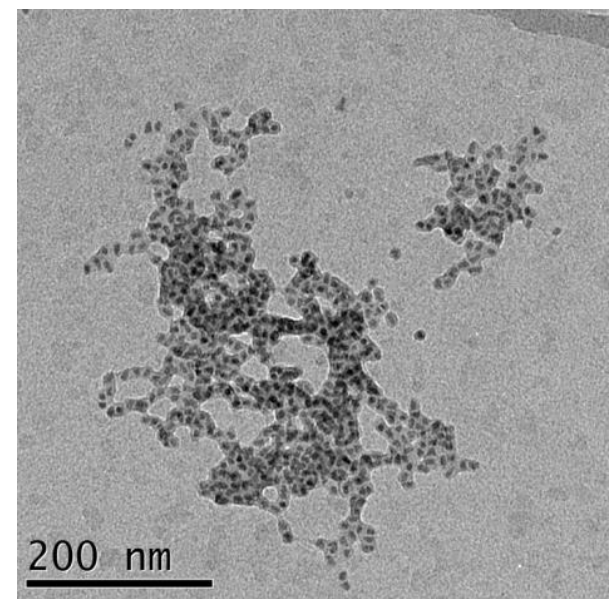

Fig. 2. (a) Cryo-TEM image of a quantum dot aggregate in a water dispersion. (b) TEM image of the same group of quantum dots after the grid has been warmed to room temperature. 